\title{
L'iposurrenalismo in pazienti che assumono glucocorticoidi per via inalatoria è molto frequente e la determinazione dei livelli di cortisolo al mattino può guidarne la gestione
}

\author{
Salvatore Cannavò ${ }^{1}$
}

Pubblicato online: 16 ottobre 2015

(C) Springer International Publishing AG 2015

Commento a:

Adrenal suppression in patients taking inhaled glucocorticoids is highly prevalent and management can be guided by morning cortisol.

C.P. Woods, N. Argese, M. Chapman, C. Boot, R. Webster, V. Dabhi, A. Grossman, A.A. Toogood, W. Arlt, P.M. Stewart, R.K. Crowley, J.W. Tomlinson. Eur J Endocrinol (2015) pii: EJE-15-0608

L'inibizione funzionale dell'asse ipotalamo-ipofisi-surrene (HPA) è un effetto indesiderato comune, anche se sottostimato, dell'assunzione cronica di glucocorticoidi, che necessita una tempestiva diagnosi e un'adeguata sostituzione ormonale. Di fatto, non esistono fattori predittivi codificati e affidabili dell'insorgenza di iposurrenalismo centrale conseguente a terapia con glucocorticoidi, condizione che può manifestarsi indipendentemente dalla tipologia e dalla via di somministrazione del farmaco, o dalla posologia e dalla durata del trattamento, sebbene si associ più frequentemente all'impiego di alte dosi e a trattamenti prolungati. Ad ogni modo, una dose di $5 \mathrm{mg}$ di prednisolone (o equivalente) assunta per via orale o parenterale è considerata potenzialmente in grado di sopprimere l'asse HPA. Al contrario, l'effetto sulla funzione surrenale di glucocorticoidi assunti per via inalatoria, topica o intra-articolare è poco noto. Nel sospetto di iposurrenalismo centrale iatrogeno, il test di stimolo con ACTH rappresenta il gold standard per la valutazione funzionale dinamica dell' asse HPA, ma uno screening sistematico dei pazienti a rischio è improponibile, in considerazione dell'elevato numero di individui che praticano terapia

$凶$ S. Cannavò

cannavos@unime.it

1 Dipartimento di Medicina Clinica e Sperimentale, Università di Messina, Messina, Italia steroidea, dei costi e della necessità di disporre di una rete di centri specialistici in grado tanto di eseguire quanto di interpretare questo test. Pertanto, sembra necessaria la ricerca di nuove strategie per l'individuazione dei pazienti a rischio.

Recentemente, Woods et al. hanno valutato la prevalenza di iposurrenalismo centrale in pazienti sottoposti a terapia con glucocorticoidi, verificando l'utilità del dosaggio del cortisolo sierico al mattino come test di screening. In questo studio retrospettivo monocentrico, il 33,2\% dei 404 pazienti in trattamento con corticosteroidi per via orale, inalatoria, nasale o topica per patologie non endocrine presenta una risposta al test di stimolo con ACTH ( $250 \mu \mathrm{g}$ i.v. in bolo) compatibile con una condizione di insufficienza surrenalica. Inoltre, il $20,5 \%$ dei pazienti in terapia con corticosteroidi per via inalatoria dimostra una risposta patologica al test con ACTH, e la prevalenza dell'insufficienza surrenale correla positivamente con la dose assunta. Nei pazienti in trattamento con corticosteroidi per via inalatoria, l'analisi delle curve ROC dimostra che un valore di cortisolo sierico al mattino $\geq 348 \mathrm{nmol} / \mathrm{l}$ ha una specificità del $100 \%$ nel predire una risposta fisiologica all' ACTH test, mentre un cortisolo $\leq 34 \mathrm{nmol} / \mathrm{l}$ ha una sensibilità del $100 \%$ nel predire una risposta patologica al test. Utilizzando questi cut-off, il test con ACTH non sarebbe stato necessario nel 50\% dei casi.

Gli autori hanno dunque dimostrato un'elevata prevalenza di insufficienza surrenale in pazienti trattati con glucocorticoidi, anche se assunti solo per via inalatoria (specie se ad alte dosi). Inoltre, questo studio dimostra che, in questo contesto, un dosaggio del cortisolo sierico al mattino può essere utile per selezionare i pazienti che necessitano ulteriori approfondimenti diagnostici (mediante test di stimolo con ACTH) per confermare o escludere una condizione di iposurrenalismo centrale iatrogeno. 